\title{
Acknowledgment of Reviewers, 1997
}

The success of PHYTOPATHOLOGY depends on the quality of manuscripts submitted by authors and on the care and competence with which they are reviewed. It is the policy of the Editorial Board to solicit reviews for manuscripts from specialists who are most qualified to review them. In addition to members of the Editorial Board, the individuals listed below provided constructive critical reviews of one or more manuscripts during the past year. Their names are published here in grateful appreciation of their contribution to the journal and to the science of phytopathology. Publication no. P-1997-1124-01O

Adams, G. C., Michigan State University, East Lansing, MI

Adaskaveg, J., University of California, Riverside, CA

Allen, C., University of Wisconsin, Madison, WI

Allison, R. F., Michigan State University, East Lansing, MI

Amerson, H., North Carolina State University, Raleigh, NC

Anderson, E., University of arkansas, Fayetteville, AR

Anderson, J., Universtiy of Toronto, Mississauga, ON, CANADA

Andrivon, D., INRA, Le Rheu, FRANCE

Aylor, D., The Connecticut Agricultural Experiment Station,

New Haven, CT

Backhouse, D., The University of Sydney, Sydney, AUSTRALIA

Bailey, B. A., USDA ARS, Beltsville, MD

Barnett, O. W., North Carolina State University, Raleigh, NC

Bauer, D., Cornell University, Ithaca, NY

Bauer, W. D., The Ohio State University, Columbus, OH

Beaver, J., University of Puerto Rico, Mayaguez, PUERTO RICO

Becker, O., University of California, Riverside, CA

Bender, C., Oklahoma State University, Stillwater, OK

Benson, M., North Carolina State University, Raleigh, NC

Bills, G., Merck Research Laboratories, Rahway, NJ

Black, L. L., AVRDC, Shanhua, TAIWAN

Blanchette, R., University of Minnesota, St. Paul, MN

Bockus, W., Kansas State University, Manhattan, KS

Bostock, R., University of California, Davis, CA

Boudreau, M., Warren Wilson College, Asheville, NC

Bowers, J., USDA ARS, Beltsville, MD

Braun, E., Iowa State University, Ames, IA

Brigham, L., University of Arizona, Tucson, AZ

Brown, J., John Innes, Norwich, ENGLAND, UK

Brown, J. K., University of Arizona, Tucson, AZ

Brown, R., USDA ARS, New Orleans, LA

Burpee, L., University of Georgia, Griffin, GA

Burr, T., New York State Agricultural Research Station, Geneva, NY

Bushnell, W., University of Minnesota, St. Paul, MN

Cadori, R., University of Georgia, Athens, GA

Campbell, C. L., North Carolina State University, Raleigh, NC

Candresse, T., INRA, Villenauve D’Ornon, FRANCE

Carris, L., Washington State University, Pullman, WA

Cary, J., USDA ARS, New Orleans, LA

Chase, T. E., University of South Dakota, Brookings, SD

Chatterjee, A., University of Missouri, Columbia, MO

Chen, T. A., Rutgers University, New Brunswick, NJ

Chen, W., Illinois Natural History Survey, Champaign, IL

Chen, X., Washington State University, Pullman, WA

Ciuffetti, L., Oregon State University, Corvallis, OR

Clough, S. J., University of Illinois, Urbana, IL

Correll, J., University of Arkansas, Fayetteville, AR

Cubeta, M., North Carolina State University, Plymouth, NC

Culver, J., University of Maryland, College Park, MD

Cuppels, D. A., Agriculture and Agri-Food Canada, London, ON, CANADA

D'Arcy, C., University of Illinois, Urbana, IL

Daugherty, M., Cornell University, Riverhead, NY

Davis, E. L., North Carolina State University, Raleigh, NC

Davis, K., The Ohio State University, Columbus, $\mathrm{OH}$

Davis, M. J., University of Florida, Homestead, FL

Davis, R. E., USDA, Beltsville, MD

Dawson, W. O., University of Florida, Lake Alfred, FL

Deacon, J., University of Edinburgh, Edinburgh, SCOTLAND, UK

De Boer, S., Centre for Anumal and Plant Health, Prince Edward Island, CANADA
Denny, T., University of Georgia, Athens, GA

Deom, C. M., University of Georgia, Athens, GA

De Zoeten, G. A., Michigan State University, East Lansing, MI

Dion, P., University Laval, QC, CANADA

Dodds, J. A., University of California, Riverside, CA

Dolja, V., Oregon State University, Corvallis, OR

Domier, L., University of Illinois, Urbana, IL

Doudrick, R., USDA Forest Service, Saucier, MS

Drenth, A., University of Queensland, Brisbane, AUSTRALIA

Elias, K., USDA ARS, Beltsville, MD

Ellingboe, A., University of Wisconisn, Madison, WI

Elliott, M. L., University of Florida, Ft. Lauderdale, FL

English, J., University of Missouri, Columbia, MO

Eplee, R., USDA, APHIS, Oxford, NC

Everts, K., University of Delaware, Georgetown, DE

Fang, E., Clemson University, Clemson, SC

Farrar, J., Weber State University, Ogden, UT

Ferrandino, F., The Connecticut Agricultural Experiment Station, New Haven, CT

Ferris, V., Purdue University, West Layfayette, IN

Finckh, M., Gruppe Phytomedizin Pathologie, Zurich, SWITZERLAND

Fitt, B., Rothamstead Experiment Station, Harpenden, ENGLAND, UK

Fletcher, J., Oklahoma State University, Stillwater, OK

Fraedrich, S., USDA Forest Service, Athens, GA

Francis, D. M., The Ohio State University, Wooster, OH

Francl, L., North Dakota State University, Fargo, ND

Fravel, D. R., USDA ARS, Beltsville, MD

Fredriksen, R., Texas A\&M University, College Station, TX

Fulbright, D., Michigan State University, East Lansing, MI

Gabriel, D., University of Florida, Gainesville, FL

Gaudet, D. A., Agriculture Canada Research Station, Lethbridge, AB, CANADA

Geider, K., Max-Planck-Institut fur Zellbiologie, Ladenburg, GERMANY

German, T. L., University of Wisconsin, Madison, WI

Ghabrial, S., University of Kentucky, Lexington, KY

Gibson, G., University of Edinburgh, Edinburgh, SCOTLAND, UK

Gilbert, J., Agriculture and Agri-Food Canada, Winnipeg, MB, CANADA

Gilchrist, D., University of California, Davis, CA

Gitaitis, R., Coastal Plains Experimental Station, Tifton, GA

Gold, S., University of Georgia, Athens, GA

Gonzalez, C. F., Texas A\&M University, College Station, TX

Goodman, R., University of Wisconsin, Madison, WI

Goodwin, S., Purdue University, West Lafayette, IN

Gordon, T. R., University of California, Davis, CA

Gottwald, T., USDA ARS, Orlando, FL

Govers, F., Wageningen Agricultural University, Wageningen, NETHERLANDS

Gray, S. M., Cornell University, Ithaca, NY

Grisham, M. P., USDA ARS, Houma, LA

Groth, J. R., University of Minnesota, St. Paul, MN

Guest, D., University of Melbourne, Parkville, AUSTRALIA

Gulya, T., USDA, Fargo, ND

Gundersen, R. D., USDA, Beltsville, MD

Hadidi, A. F., USDA ARS, Beltsville, MD

Halbrendt, J., Pennsylvania State University, Bigleville, PA

Hammerschmidt, R., Michigan State University, East Lansing, MI

Hammond, J., USDA, Beltsville, MD

4 PHYTOPATHOLOGY 
Handelsman, J., University of Wisconsin, Madison, WI

Hanlin, R. T., University of Georgia, Athens, GA

Hansen, E. M., Oregon State University, Corvallis, OR

Harman, G. E., New York State Agricultural Research Station, Geneva, NY

Harrington, T., Iowa State University, Ames, IA

Harrison, N., University of Florida, Ft. Lauderdale, FL

Hartung, J., USDA ARS, Beltsville, MD

Hayes, P., Oregon State University, Corvallis, OR

Hayward, A. C., University of Queensland, Brisbane, AUSTRALIA

He, S. Y., Michigan State University, East Lansing, MI

Heaton, L., Kansas State University, Manhattan, KS

Helgeson, J., University of Wisconsin, Madison, WI

Henson, J., Montana State University, Bozeman, MT

Hickey, K., Pennsylvania State University, Biglerville, PA

Higgins, V., Universtiy of Toronto,Totonto, ON, CANADA

Hirano, S., University of Wisconsin, Madison, WI

Hoitink, H., The Ohio State University, Wooster, OH

Hood, M., Duke University, Durham, NC

Hopkins, D., University of Florida, Leesburg, FL

Hoy, J., Louisiana State University, Baton Rouge, LA

Huber, D., Purdue University, West Lafayette, IN

Hughes, G., University of Edinburgh, Edinburgh, SCOTLAND, UK

Hunt, A. G., University. of Kentucky, Lexington, KY

Hussey, R., University of Georgia, Athens, GA

Jaboaji-Hare, S., Macdonald Campus of McGill University,

Ste. Anne Bellevue, QC, CANADA

Janisiewicz, W. J., USDA ARS, Kearneysville, WV

Jarosz, A., Michigan State University, East Lansing, MI

Jeffers, S. N., Clemson University, Clemson, SC

Jin, Y., South Dakota State University, Brookings, SD

Johnson, K. B., Oregon State University, Corvallis, OR

Jones, A. L., Michigan State University, East Lansing, MI

Judelson, H., University of California, Riverside, CA

Kaplan, D., USDA, Orlando, FL

Karasev, A., University of Florida, Lake Alfred, FL

Katan, J., The Hebrew University of Jerusalem, Rehovot, ISRAEL

Kelly, J. D., Michigan State University, East Lansing, MI

Kirby, G. C., Flinders University, Adelaide, AUSTRALIA

Kirpatrick, B., University of California, Davis, CA

Kistler, H. C., University of Florida, Gainesville, FL

Kloepper, J. W., Auburn University, Auburn, AL

Kluepfel, D., Clemson University, Clemson, SC

Kobayashi, D. Y., Rutgers University, New Brunswick, NJ

Kohn, L., University of Toronto, Mississauga, ON, CANADA

Kolmer, J., Agriculture Canada Research Station, Winnipeg, MB, CANADA

Kraft, J. M., USDA ARS, Prosser, WA

Kuhn, D., Florida International University, Miama, FL

Lacy, G. H., Virginia Polytechnic Institute and State University, Blacksburg, VA

Lannou, C., INRA, Thiverval-Grignon, FRANCE

Larkin, R. P., USDA ARS, Beltsville, MD

Larsen, M., Forest Sciences Laboratory, Moscow, ID

Laurence, J., Boyce Thompson Institute, Ithaca, NY

Lawrence, D. M., University of California, Berkeley, CA

Leath, S., North Carolina State University, Raleigh, NC

Lee, I.-M., USDA ARS, Beltsville, MD

Lefevre, F., INRA, Avignon, FRANCE

Legard, D. E., University of Florida, Dover, FL

Levesque, C. A., Agriculture \& Agri-Food Canada, Summerland, BC, CANADA

Lewis, K., University of Northern British Columbia, Prince George, BC, CANADA

Lim, S. M., University of Arkansas, Fayetteville, AR

Linderman, R. G., USDA ARS, Corvallis, OR

Lindow, S., University of California, Berkeley, CA

Lindsey, D., New Mexico State University, Las Cruces, NM

Line, R. F., Washington State University, Pullman, WA

Lipps, P., The Ohio State University, Wooster, OH

Liu, J. Q., Agriculture and Agri-Food Canada, Winnipeg, MB, CANADA

Livingston, W., University of Maine, Orono, ME

Lommel, S. A., North Carolina State University, Raleigh, NC

Loper, J., USDA ARS, Corvallis, OR
Lopes, D. B., University of Florida, Gainesville, FL

Louws, F., North Carolina State University, Raleigh, NC

MacHardy, W. E., University of New Hampshire, Durham, NH

Macko, S., University of Virginia, Charlottesville, VA

Madden, L. V., The Ohio State University, Wooster, OH

Mahaffee, W., USDA ARS, Corvallis, OR

Manning, B., University of Massachusetts, Amherst, MA

Marchetti, M., USDA ARS, Beaumont, TX

Marchetti, T., USDA ARS, Beaumont, TX

Marco, Y., CNRS-INRA, Tolosan Cedex, FRANCE

Marois, J. J., University of Florida, Quincy, FL

Martin, F. N., USDA ARS, Salinas, CA

Maule, A., John Innes Institute, Norwich, ENGLAND, UK

May, G., University of Minnesota, St. Paul, MN

Mayo, M., Scottish Crop Research Institute, Invergowrie, SCOTLAND, UK

McCormick, S., USDA ARS, Peoria, IL

McDonald, B., Texas A\&M University, College Station, TX

McK. Bird, D., North Carolina State University, Raleigh, NC

McNabb, H. S., Jr., Iowa State University, Ames, IA

Menge, J., University of California, Riverside, CA

Michailides, T., University of California, Parlier, CA

Milgroom, M. G., Cornell University, Ithaca, NY

Miller, D., Agriculture \& Agri-Food Canada, Ottawa, ON, CANADA

Miller, S., The Ohio State University, Wooster, OH

Miller, W. A., Iowa State University, Ames, IA

Mink, G., Washington State University, Prosser, WA

Money, N. P., Miami University, Oxford, $\mathrm{OH}$

Moore, L., Oregon State University, Corvallis, OR

Morris, T. J., University of Nebraska, Lincoln, NE

Moyer, J. W., North Carolina State University, Raleigh, NC

Mundt, C., Oregon State University, Corvallis, OR

Munkvold, G., Iowa State University, Ames, IA

Murphy, J., Auburn University, Auburn, AL

Murray, T. D., Washington State University, Pullman, WA

Mussleman, L., Old Dominion University, Norfolk, VA

Nault, L. R., The Ohio State University, Wooster, OH

Neate, S., CSIRO, Glen Osmond, AUSTRALIA

Neher, D., University of Toledo, Toledo, $\mathrm{OH}$

Nelson, E. B., Cornell University, Ithaca, NY

Nelson, R. S., Noble Foundation, Ardmore, OK

Newcombe, G., Washington State University, Puyallup, WA

Niblack, T., University of Missouri, Columbia, MO

Nicholson, R., Purdue University, West Lafayette, IN

Nutter, F. W., Iowa State University, Ames, IA

Oudemans, P., Rutgers University, Chatsworth, NJ

Panaccione, D., West Virginia University, Morgantown, WV

Pataky, J., University of Illinois, Urbana, IL

Petty, I. T. D., North Carolina State University, Raleigh, NC

Pierce, M., Oklahoma State University, Stillwater, OK

Pierson, L. S., University of Arizona, Tucson. AZ

Pinkerton, J. N., USDA ARS Hort. Crops Research Lab, Corvallis, OR

Pirone, T., University of Kentucky, Lexington, KY

Powers, T. O., University of Nebraska, Lincoln, NE

Pruvost, O., CIRAD FLHOR, Reunion Island, FRANCE

Pueppke, S., University of Missouri, Columbia, MO

Punja, Z. K., Simon Fraser University, Burnaby, BC, CANADA

Pusey, P. L., USDA ARS, Wenatchee, WA

Quigley, N. B., University of Tennessee, Knoxville, TN

Raaijmakers, J., USDA ARS, Pullman, WA

Reeleder, R. D., Agriculture Canada Research Station, Delhi, ON, CANADA

Ritchie, D. F., North Carolina State University, Raleigh, NC

Roberts, D., USDA ARS, Beltsville, MD

Roberts, R. G., USDA ARS, Wenatchee, WA

Rochon, D. M., Agriculture and Agri-Food Canada, Summerland, BC, CANADA

Roncadori, R., University of Georgia, Athens, GA

Roossinck, M. J., Noble Foundation, Ardmore, OK

Rothrock, C. S., University of Arkansas, Fayetteville, AR

Rouse, D. I., University of Wisconsin, Madison, WI

Rowe, R. C., The Ohio State University, Wooster, $\mathrm{OH}$

Rufty, R. C., North Carolina State University, Raleigh, NC

Rupe, J. C., University of Arkansas, Fayetteville, AR

Rush, M. C., Louisiana State University, Baton Rouge, LA 
Samac, D. A., USDA ARS, St. Paul, MN

Sarah, J. L., CIRAD FLHOR, Montpellier Cedex, FRANCE

Savary, S. S., IRRI, Manila, PHILIPPINES

Schardl, C. L., University of Kentucky, Lexington, KY

Scherm, H., University of Georgia, Athens, GA

Schisler, D. A., USDA ARS, Peoria, IL

Schneider, R. W., Louisiana State University, Baton Rouge, LA

Schoelz, J., University of Missouri, Columbia, MO

Scholthof, H. B., Texas A\&M University, College Station, TX

Scholthof, K.-B., Texas A\&M University, College Station, TX

Schottel, J. L., University of Minnesota, St. Paul, MN

Scott-Craig, J., Michigan State University, East Lansing, MI

Seem, B., New York State Agricultural Research Station, Geneva, NY

Senior, L., North Carolina State University, Raleigh, NC

Shaner, G. E., Purdue University, West Lafayette, IN

Shaw, M., New Mexico Hgihlands University, Las Vegas, NM

Shaw, M. W., University of Reading, Reading, ENGLAND, UK

Shelby, R., Auburn University, Auburn University, AL

Sherwood, J. E., Montana State University, Bozeman, MT

Sherwood, J. L.,University of Georgia, Athens, GA

Simcox, K., The Ohio State University, Wooster, OH

Sinclair, W. A., Cornell University, Ithaca, NY

Singh, R. P., CIMMYT, Mexico, D.F., MEXICO

Smith, B., USDA ARS, Poplarville, MS

Smith, C. A., University of New Hampshire, Durham, NH

Sneh, B., Tel Aviv University, Tel Aviv, ISRAEL

Snetselaar, K., St. Josephs University, Philadelphia, PA

Spotts, B., Mid-Columbia AREC, Hood River, OR

Stall, R. E., University of Florida, Gainesville, FL

Stanghellini, M. E., University of California, Riverside, CA

Stapleton, J., Kearney Agricultural Center, Parlier, CA

Starr, J. L., Texas A\&M University, College Station, TX

Stavely, J. R., USDA ARS, Beltsville, MD

Steffenson, B. J., North Dakota State University, Fargo, ND

Stenger, D., USDA ARS, Lincoln, NE

Stevens, M. R., Brigham Young University, Provo, UT

Stockwell, V. O., USDA ARS, Corvallis, OR
Stromberg, E. L., Virginia Polytechnic Institute and State University, Blacksburg, VA

Stuteville, D. L., Kansas State University, Manhattan, KS

Taylor, J., Stephen F. Austin State University, Nacogdoches, TX

TeBeest, D., University of Arkansas, Fayetteville, AR

Teviotdale, B., Kearny Agricultural Center, Parlier, CA

Thomas, C., USDA ARS, Charleston, SC

Timmer, L. W., University of Florida, Lake Alfred, FL

Tooley, P., USDA ARS, Frederick, MD

Tumer, N. E., Rutgers University, New Brunswick, NJ

Tuzun, S., Auburn University, Auburn, AL

Ullman, D. E., University of California, Davis, CA

Upper, C., USDA ARS, Madison, WI

Vaillancourt, L., University of Kentucky, Lexington, KY

Van Bruggen, A., University of California, Davis, CA

Van Gijsegem, F., CNRS-INRA, Tolosan Cedex, FRANCE

Van Loon, L. C., Utrecht University, Utrecht, NETHERLANDS

Vanneste, J. L., Ruakura Research Centre, Hamilton, NEW ZEALAND

Vidaver, A., University of Nebraska, Lincoln, NE

Voelksch, B., Friedrich Schiller Universitaet Jena, Jena, GERMANY

VonBroembsen, S. L., Oklahoma State University, Stillwater, OK

Weeden, N. F., New York State Agricultural Experiment Station, Geneva, NY

Weiland, J., USDA ARS, Fargo, ND

White, J. F., Jr., Rutgers University, New Brunswick, NJ

Wick, R., University of Massachusetts, Amherst, MA

Wilcox, W., New York State Agricultural Research Station, Geneva, NY

Williams, P., University of Wisconsin, Madison, WI

Willis, D. K., University of Wisconsin, Madison, WI

Wilson, D. M., Coastal Plains Experimental Station, Tifton, GA

Wingfield, B., University of the Orange Free State, Bloemfontein, SOUTH AFRICA

Woloshuk, C., Purdue University, West Lafayette, IN

Yang, X.-B., Iowa State University, Ames, IA

Yassi, M., University of California, Riverside, CA

Young, M., Montana State University, Bozeman, MT

Zeller, K., Kansas State University, Manhattan, KS

Zook, M., Michigan State University, East Lansing, MI 